\title{
Boundary Problems with Meromorphic Symbols in Cylindrical Domains
}

\author{
G. Harutjunjan and B.-W. Schulze
}

August 5, 2003

\begin{abstract}
We show relative index formulas for boundary value problems in cylindrical domains and Sobolev spaces with different weights at $\pm \infty$. The amplitude functions are meromorphic in the axial covariable and take values in the space of boundary value problems on the cross section of the cylinder.
\end{abstract}

AMS CLASSIFICATION: 35J40, 47G30, 58J32.

\section{Introduction}

This paper is aimed at studying boundary value problems in an infinite cylinder $\mathbb{R} \times X$, where $X$ is a compact $C^{\infty}$ manifold with boundary $Y$. The operators on $X$ will be represented in terms of amplitude functions with respect to the axial variable $t$ and a complex covariable $w$ as meromorphic functions in the complex $w$-plane taking values in the algebra of pseudo-differential boundary value problems on the cross section $X$. We study ellipticity and Fredholm property in (doubly) weighted Sobolev spaces and express parametrices within the calculus as well as the relative index with respect to changes of weights. Problems of a similar structure including relative index results are well known for the case of a closed compact cross section $X$, cf. Melrose and Mendoza [9], Schulze and Tarkhanov [14]. Boundary problems for differential operators in weighted Sobolev spaces in infinite cylinders have been systematically studied in Sternin [15].

Results of a similar type in the pseudo-differential set-up are necessary in mixed elliptic problems, as they are studied in [2], and also in other interesting new applications with geometric singularities, e.g., crack problems, cf. Kapanadze and Schulze [6]. This is the main motivation of the present investigation. The main results are Theorems 2.8 and 2.9, and the conclusions are explicitly applied in [2]. Let us also note that meromorphic families of boundary value problems are studied in connection with operators on manifolds with conical singularities, cf. Kondratyev [7], Schrohe and Schulze [11], [12] or, in the context of long-time asymptotics for the parabolic case Krainer [8].

As far as we employ background from [14] or Gohberg and Sigal [3] we will content ourselves with references. An inspection of the methods shows that it is essential to have transposed operators in the calculus. Those are not always available in boundary value problems with the transmission property, namely when the operators have non-vanishing type or order (which is the case for differential boundary problems). One of the new points in this paper is to avoid this difficulty by applying a specific class of holomorphic reductions 
of orders, see Harutjunjan and Schulze [5] which do not contribute to zeros and poles in a prescribed weight strip.

One also should possess results of that kind in more general situations, e.g., when the cross sections have geometric singularities and boundary. In this sense another consequence of our investigation is a general and transparent approach which makes it easy to predict similar relative index results also for cross sections with higher singularities, cf [4].

\section{Meromorphic symbolic calculus}

\subsection{Boundary value problems with parameters}

If $M$ is a closed compact $C^{\infty}$ manifold by $L_{\mathrm{cl}}^{\mu}\left(M ; \mathbb{R}^{l}\right)$ we denote the space of all classical pseudo-differential operators of order $\mu \in \mathbb{R}$ on $M$ depending on a parameter $\lambda \in \mathbb{R}^{l}$. More precisely, the local amplitude functions $a(x, \xi, \lambda)$ belong to the standard classical symbol spaces

$$
S_{\mathrm{cl}}^{\mu}\left(U \times \mathbb{R}_{\xi, \lambda}^{n+l}\right)
$$

$U \subseteq \mathbb{R}^{n}$ open, $n=\operatorname{dim} M, L^{-\infty}\left(M ; \mathbb{R}^{l}\right):=\mathcal{S}\left(\mathbb{R}^{l}, L^{-\infty}(M)\right)$ with $L^{-\infty}(M) \cong C^{\infty}(M \times M)$ being the space of smoothing operators on $M$, and $\mathcal{S}\left(\mathbb{R}^{l}, \ldots\right)$ the Schwartz space with values in the space in the brackets. Throughout this exposition all manifolds in question are assumed to be equipped with Riemannian metrics. This gives us $L^{2}$ - spaces with a fixed scalar product (in the compact case, otherwise local $L^{2}$-spaces). Moreover, $H^{s}(M)$ denotes the scale of standard Sobolev spaces on $M$ of smoothness $s \in \mathbb{R}$. It will be convenient to formulate our consideration in terms of distributional sections $H^{s}(M, E)$ in smooth complex vector bundles $E$, all assumed to be equipped with Hermitian metrics. Let Vect $(\cdot)$ denote the set of all smooth complex vector bundles on the manifold in the brackets. If $X$ is a compact $C^{\infty}$ manifold with boundary we set $H^{s}(\operatorname{int} X, E):=\left.H^{s}(2 X, \tilde{E})\right|_{\operatorname{int} X}$ for an $\tilde{E} \in \operatorname{Vect}(2 X)$ and $E:=\left.\tilde{E}\right|_{\text {int } X}$; here $2 X$ denotes the double of $X$, obtained by gluing together two copies $X_{-}$ and $X_{+}$of $X$ along the common boundary (where we identify $X_{+}$with $X$ ).

Let us now pass to the space $\mathcal{B}^{\boldsymbol{\mu}, \mathrm{d}}\left(X ; \boldsymbol{v} ; \mathbb{R}^{l}\right)$ of parameter-dependent boundary value problems for a compact $C^{\infty}$ manifold $X$ with boundary $Y ; \boldsymbol{\mu}, \mathrm{d}$ and $\boldsymbol{v}$ will be explained below. The operators are assumed to be of the form

$$
\mathcal{A}: \underset{\substack{\oplus_{k=1}^{K} H^{s-\frac{1}{2}-\nu_{k}}\left(Y, J_{k}\right) \\ \bigoplus_{j=1}(\operatorname{int} X, E)}}{\bigoplus_{j=1}^{J} H^{s-\frac{1}{2}-\rho_{j}}\left(Y, G_{j}\right)}
$$

for $E, F \in \operatorname{Vect}(X), J_{k}, G_{j} \in \operatorname{Vect}(Y)$, and we set $\boldsymbol{v}:=\left(E, F ;\left(J_{k}\right)_{k=1, \ldots, K},\left(G_{j}\right)_{j=1, \ldots, J}\right), \boldsymbol{\mu}=$ $\left(\begin{array}{cc}\mu & \left(\mu-\frac{1}{2}-\nu_{k}\right)_{1 \leq k \leq K} \\ { }^{\mathrm{t}}\left(\rho_{j}+\frac{1}{2}\right)_{1 \leq j \leq J} & \left(\rho_{j}-\nu_{k}\right)_{\substack{1 \leq j \leq J \\ 1 \leq k \leq K}}\end{array}\right)$. Concerning the admitted $s$ we assume that $\mathcal{A}$ is of type $\mathrm{d} \in \mathbb{N}$ and take $s>\mathrm{d}-\frac{1}{2}$. Let us write $\mathcal{A}$ as a block matrix

$$
\mathcal{A}=\left(\begin{array}{ll}
A & \mathcal{K} \\
\mathcal{T} & \mathcal{Q}
\end{array}\right)
$$

with $A(\lambda): H^{s}(\operatorname{int} X, E) \rightarrow H^{s-\mu}(\operatorname{int} X, F)$ in the upper left corner, cf. the formula (5) below. The lower right $\mathcal{Q}(\lambda)$ is assumed to be an $J \times K$ matrix of elements in $L_{\mathrm{cl}}^{\mu_{j k}}\left(Y ; J_{k}, G_{j} ; \mathbb{R}^{l}\right)$ with Douglis-Nirenberg orders $\mu_{j k}=\rho_{j}-\nu_{k}$ for certain $\rho_{j}, \nu_{k} \in \mathbb{R}$. The components of 
$\mathcal{T}(\lambda)={ }^{\mathrm{t}}\left(T_{1}(\lambda), \ldots, T_{J}(\lambda)\right)$ are so called (parameter-dependent) trace operators of type $\mathrm{d} \in \mathbb{N}$ in Boutet de Monvel's calculus, ord $T_{j}=\rho_{j}+\frac{1}{2}$, and $\mathcal{K}(\lambda)=\left(K_{1}(\lambda), \ldots, K_{K}(\lambda)\right)$ is a vector of potential operators $K_{k}(\lambda)$ of order $\mu-\frac{1}{2}-\nu_{k}$.

The operators $\mathcal{A} \in \mathcal{B}^{\boldsymbol{\mu}, \mathrm{d}}\left(X ; \boldsymbol{v} ; \mathbb{R}^{l}\right)$ have a (parameter-dependent) principal symbolic structure

$$
\sigma(\mathcal{A})=\left(\sigma_{\psi}(\mathcal{A}), \sigma_{\partial}(\mathcal{A})\right)
$$

consisting of the homogeneous (in $(\xi, \lambda) \neq 0$ ) principal interior symbol

$$
\sigma_{\psi}(\mathcal{A}):=\sigma_{\psi}(A): \pi_{X}^{*} E \rightarrow \pi_{X}^{*} F
$$

with the canonical projection $\pi_{X}: T^{*} X \times \mathbb{R}^{l} \backslash 0 \rightarrow X(0$ means here $(\xi, \lambda)=0)$ and the homogeneous (in $(\eta, \lambda) \neq 0$ ) principal boundary symbol

$$
\sigma_{\partial}(\mathcal{A}): \pi_{Y}^{*}\left(\begin{array}{c}
E^{\prime} \otimes H^{s}\left(\mathbb{R}_{+}\right) \\
\oplus \\
\oplus_{k=1}^{K} J_{k}
\end{array}\right) \rightarrow \pi_{Y}^{*}\left(\begin{array}{c}
F^{\prime} \otimes H^{s-\mu}\left(\mathbb{R}_{+}\right) \\
\oplus \\
\oplus_{j=1}^{J} G_{j}
\end{array}\right)
$$

with the canonical projection $\pi_{Y}: T^{*} Y \times \mathbb{R}^{l} \backslash 0 \rightarrow Y(0$ in this case means $(\eta, \lambda)=0)$, $s>\mathrm{d}-\frac{1}{2}, E^{\prime}:=\left.E\right|_{Y}, F^{\prime}:=\left.F\right|_{Y}$.

Definition 1.1 An element $\mathcal{A} \in \mathcal{B}^{\boldsymbol{\mu}, \mathrm{d}}\left(X ; \boldsymbol{v} ; \mathbb{R}^{l}\right)$ is said to be parameter-dependent elliptic if both (3) and (4) are isomorphisms (in (4) this may be required for some fixed $s>\max (\mu, d)-$ $\left.\frac{1}{2}\right)$.

To describe the operators $A$ in (1) we denote by $L_{\mathrm{cl}}^{\mu}\left(2 X ; \tilde{E}, \tilde{F} ; \mathbb{R}^{l}\right)_{\operatorname{tr}}$ the subspace of all elements of $L_{\mathrm{cl}}^{\mu}\left(2 X ; \tilde{E}, \tilde{F} ; \mathbb{R}^{l}\right)$ that have the transmission property at the boundary $Y$; concerning the definition on the level of local symbols and further details cf. Boutet de Monvel [1] or Rempel and Schulze [10]. For references below we give an outline on some elements of the calculus.

The upper left corner $A$ of (1) has the form

$$
A(\lambda)=\mathrm{r}^{+} P(\lambda) \mathrm{e}^{+}+G(\lambda)
$$

for an element $P(\lambda)$ of $L_{\mathrm{cl}}^{\mu}\left(2 X ; \tilde{E}, \tilde{F} ; \mathbb{R}^{l}\right)_{\mathrm{tr}}, E=\left.\tilde{E}\right|_{X}, F=\left.\tilde{F}\right|_{X} ; \mathrm{e}^{+}$is the operator of extension by zero from int $X$ to $2 X$ and $\mathrm{r}^{+}$the restriction from $2 X$ to int $X$. The second summand $G(\lambda)$ is a family of so called Green operators. Let us recall the structure of $G(\lambda)$ together with the trace and potential operators. We will formulate the elements of the calculus for the case $\mu \in \mathbb{Z}$ and $\rho_{j}=\mu, \nu_{k}=0$ for all $j, k$ and then write $\mathcal{B}^{\mu, \mathrm{d}}$ instead of $\mathcal{B}^{\mu, \mathrm{d}}$. By using reductions of orders on the boundary with a suitable holomorphic dependence of a complex parameter, cf. Theorem 1.5 below, we then obtain all results also for the general case.

By $\mathcal{B}^{-\infty, 0}\left(X ; \boldsymbol{v} ; \mathbb{R}^{l}\right)$ for $\boldsymbol{v}=(E, F ; J, G), J:=\oplus_{k=1}^{K} J_{k}, G:=\oplus_{j=1}^{J} G_{k}$, we understand the space of all smoothing elements of type 0 of the calculus of boundary value problems. For $l=0$ and trivial bundles of fibre dimension 1 this simply means that the entries of $(2)$ have $C^{\infty}$ kernels on $X \times X, X \times Y, Y \times X$ and $Y \times Y$, respectively. This has a straightforward generalisation to the case of arbitrary bundles $E, F$, etc. The resulting space $\mathcal{B}^{-\infty, 0}(X ; \boldsymbol{v})$ is then Fréchet, and we set $\mathcal{B}^{-\infty, 0}\left(X ; \boldsymbol{v} ; \mathbb{R}^{l}\right):=\mathcal{S}\left(\mathbb{R}^{l}, \mathcal{B}^{-\infty, 0}(X ; \boldsymbol{v})\right)$. For $\mathrm{d} \in \mathbb{N}$ the space $\mathcal{B}^{-\infty, \mathrm{d}}\left(X ; \boldsymbol{v} ; \mathbb{R}^{l}\right)$ is the set of all operator families of the form

$$
\mathcal{G}(\lambda)=\mathcal{G}_{0}(\lambda)+\sum_{j=1}^{\mathrm{d}} \mathcal{G}_{j}(\lambda) \operatorname{diag}\left(T^{j}, 0\right)
$$


where $T$ is any first order differential operator $C^{\infty}(X, E) \rightarrow C^{\infty}(X, E)$ the homogeneous principal symbol of which near $Y$ is equal to $\xi_{n} \otimes \operatorname{id}_{\pi_{X}^{*} E}$, where $\xi_{n}$ is the covariable to $x_{n}$, the normal to $Y$ in a collar neighbourhood of the boundary.

Now let $\mathcal{B}_{G}^{\mu, 0}\left(X ; \boldsymbol{v} ; \mathbb{R}^{l}\right)$ defined to be the set of all operator families $C^{\infty}(X, E) \oplus C^{\infty}(Y, J) \rightarrow$ $C^{\infty}(X, F) \oplus C^{\infty}(Y, G)$ which are $\bmod \mathcal{B}^{-\infty, 0}\left(X ; \boldsymbol{v} ; \mathbb{R}^{l}\right)$ locally near any point of $Y$ of the form $\omega \mathrm{Op}(g) \tilde{\omega}$ for some $\omega, \tilde{\omega} \in C^{\infty}(X)$ supported in a collar neighbourhood of $Y$ and equal to 1 near $Y$ and a so called Green symbol $g(y, \eta, \lambda)$ of type 0 . By this we mean a classical operator-valued symbol

$$
g(y, \eta, \lambda) \in S_{\mathrm{cl}}^{\mu}\left(\Omega \times \mathbb{R}^{n-1} ; L^{2}\left(\mathbb{R}_{+}\right) \oplus \mathbb{C}^{j}, \mathcal{S}\left(\overline{\mathbb{R}}_{+}\right) \oplus \mathbb{C}^{j^{\prime}}\right)
$$

with $j$ and $j^{\prime}$ being the fibre dimensions of $J$ and $G$, respectively, such that the pointwise formal adjoint $g^{*}(y, \eta, \lambda)$ is of analogous structure (of course, with interchanged role of $j$ and $\left.j^{\prime}\right)$. Moreover, $\mathcal{B}_{G}^{\mu, d}\left(X ; \boldsymbol{v} ; \mathbb{R}^{l}\right)$ denotes the set of all operator families of the form (6) for arbitrary $\mathcal{G}_{j}(\lambda) \in \mathcal{B}_{G}^{\mu-j, 0}\left(X ; \boldsymbol{v} ; \mathbb{R}^{l}\right)$.

Here and in the sequel we employ material on operator-valued symbols with twisted homogeneity $\mu$. Let us briefly recall the meaning.

If $H$ is a Hilbert space and $\left\{\kappa_{\lambda}\right\}_{\lambda \in \mathbb{R}_{+}}$a strongly continuous group of isomorphisms $\kappa_{\lambda}$ : $H \rightarrow H$ with $\kappa_{\lambda} \kappa_{\lambda^{\prime}}=\kappa_{\lambda \lambda^{\prime}}$ for all $\lambda, \lambda^{\prime} \in \mathbb{R}_{+}$we say that $H$ is equipped with a group action. Let $\tilde{H}$ be a second Hilbert space with group action $\left\{\tilde{\kappa}_{\lambda}\right\}_{\lambda \in \mathbb{R}_{+}}$.

Definition 1.2 The space $S^{\mu}\left(U \times \mathbb{R}^{q} ; H, \tilde{H}\right)$ for $\mu \in \mathbb{R}, U \subseteq \mathbb{R}^{p}$ open, is defined to be the set of all $\mathcal{L}(H, \tilde{H})$-valued $C^{\infty}$ functions $a(y, \eta)$ on $U \times \mathbb{R}^{q}$ which have the property that

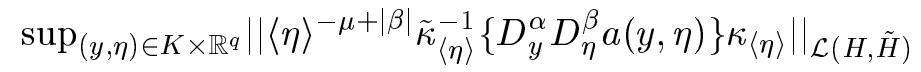

is finite for every $K \subset \subset U, \alpha \in \mathbb{N}^{p}, \beta \in \mathbb{N}^{q}$. Here $\langle\eta\rangle:=\left(1+|\eta|^{2}\right)^{\frac{1}{2}}$.

The elements of $S^{\mu}\left(U \times \mathbb{R}^{q} ; H, \tilde{H}\right)$ are called operator-valued symbols (with twisted symbolic estimates). The subspace of classical symbols $S_{\mathrm{cl}}^{\mu}\left(U \times \mathbb{R}^{q} ; H, \tilde{H}\right) \ni a(y, \eta)$ is defined by the condition that there are homogeneous components $a_{(\mu-j)}(y, \eta), j \in \mathbb{N}$ (that is $a_{(\mu-j)}(y, \lambda \eta)=\lambda^{\mu-j} \tilde{\kappa}_{\lambda} a_{(\mu-j)}(y, \eta) \kappa_{\lambda}^{-1}$ for all $(y, \eta) \in U \times\left(\mathbb{R}^{q} \backslash\{0\}\right), \lambda \in \mathbb{R}_{+}$, such that

$$
a(y, \eta) \sim \sum_{j=0}^{\infty} \chi(\eta) a_{(\mu-j)}(y, \eta)
$$

for any excision function $\chi(\eta)$ (i.e., $\chi \in C^{\infty}\left(\mathbb{R}^{q}\right), \chi(\eta)=0$ for $|\eta|<c_{0}, \chi(\eta)=1$ for $|\eta|>c_{1}$ for some constants $\left.0<c_{0}<c_{1}\right)$. The spaces $S_{(\mathrm{cl})}^{\mu}\left(U \times \mathbb{R}^{q} ; H, \tilde{H}\right)$ are Fréchet in a natural way (here we write ' $(\mathrm{cl})$ ' if a consideration is valid both for the classical and the general case).

The definitions extend to the case of Fréchet spaces $H$ or $\tilde{H}$ with group actions. For instance, let $\tilde{H}=\varliminf_{j \in \mathbb{N}} \tilde{H}^{j}$ be a projective limit of Hilbert spaces with continuous embeddings $\tilde{H}^{j+1} \hookrightarrow \tilde{H}^{j}$ for all $j$, and let $\tilde{H}^{0}$ be endowed with a group action which restricts to a group action on $\tilde{H}^{j}$ for every $j$. Then we set $S_{(\mathrm{cl})}^{\mu}\left(U \times \mathbb{R}^{q} ; H, \tilde{H}\right)=\lim _{j \in \mathbb{N}} S_{(\mathrm{cl})}^{\mu}\left(U \times \mathbb{R}^{q} ; H, \tilde{H}^{j}\right)$.

An example for the latter case is

$$
\mathcal{S}\left(\overline{\mathbb{R}}_{+}\right) \oplus \mathbb{C}^{j^{\prime}}=\lim _{j \in \mathbb{N}}\left\langle x_{n}\right\rangle^{-j} H^{j}\left(\mathbb{R}_{+}\right) \oplus \mathbb{C}^{j^{\prime}}
$$

with the group action $\kappa_{\lambda}\left(u\left(x_{n}\right) \oplus c\right):=\lambda^{\frac{1}{2}} u\left(\lambda x_{n}\right) \oplus \lambda^{ \pm \frac{1}{2}} c$ for $u \in\left\langle x_{n}\right\rangle^{-j} H^{j}\left(\mathbb{R}_{+}\right), c \in \mathbb{C}^{j^{\prime}} ;$ the plus sign is taken for (7), the minus sign for the formal adjoint. 
Theorem 1.3 Let $\mathcal{A} \in \mathcal{B}^{\boldsymbol{\mu}, \mathrm{d}}\left(X ; \boldsymbol{v} ; \mathbb{R}^{l}\right)$ be parameter-dependent elliptic. Then there exists a parametrix $\mathcal{P} \in \mathcal{B}^{-\boldsymbol{\mu},(\mathrm{d}-\mu)^{+}}\left(X ; \boldsymbol{v}^{-1} ; \mathbb{R}^{l}\right)$ in the sense that $\mathcal{I}-\mathcal{P} \mathcal{A} \in \mathcal{B}^{-\infty, \mathrm{d}_{l}}\left(X ; E, E, J, J ; \mathbb{R}^{l}\right), \mathrm{d}_{l}=$ $\max (\mu, \mathrm{d})$, and $\mathcal{I}-\mathcal{A P} \in \mathcal{B}^{-\infty,(\mathrm{d}-\mu)^{+}}\left(X ; F, F, G, G ; \mathbb{R}^{l}\right)$. Moreover, (1) is a family of Fredholm operators for any $s>\max (\mu, \mathrm{d})-\frac{1}{2}$, and there is a $C>0$ such that (1) are isomorphisms for all $|\lambda|>C$. $[6])$.

A proof may be found in [10], see also [12] or [8] (concerning an evaluation of types, see

\subsection{Meromorphic families}

In this section we introduce meromorphic families of parameter-dependent pseudo-differential operators, first for the case of a closed compact $C^{\infty}$ manifold $M$ and then on a compact $C^{\infty}$ manifold $X$ with boundary.

If $F$ is a Fréchet space and $U \subseteq \mathbb{C}$ an open set by $\mathcal{A}(U, F)$ we denote the space of all holomorphic functions in $U$ with values in $F$.

Let $L_{\mathrm{cl}}^{\mu}\left(M ; \mathbb{C} \times \mathbb{R}^{l}\right)$ denote the subspace of all operator families $\mathcal{A}(w, \lambda) \in \mathcal{A}\left(\mathbb{C}, L_{\mathrm{cl}}^{\mu}\left(M ; \mathbb{R}^{l}\right)\right)$ such that

$$
A(\tau+i \gamma, \lambda) \in L_{\mathrm{cl}}^{\mu}\left(M ; \mathbb{R}_{\tau, \lambda}^{1+l}\right)
$$

for every $\gamma \in \mathbb{R}$ and uniformly in compact $\gamma$-intervals. We will employ the following result from [8].

Theorem 1.4 For every $P(\tau, \lambda) \in L_{\mathrm{cl}}^{\mu}\left(M ; \mathbb{R}_{\tau, \lambda}^{1+l}\right)$ there exists an element $A(w, \lambda) \in L_{\mathrm{cl}}^{\mu}(M ; \mathbb{C} \times$ $\left.\mathbb{R}^{l}\right)$ such that $A(\tau, \lambda)-P(\tau, \lambda) \in L^{-\infty}\left(M ; \mathbb{R}^{1+l}\right)$. In addition, if $P(\tau, \lambda)$ is parameterdependent elliptic of order $\mu$ with the parameter $(\tau, \lambda) \in \mathbb{R}^{1+l}$, also $A(\tau+i \gamma, \lambda)$ is parameterdependent elliptic for every $\gamma \in \mathbb{R}$.

As is well known, if an operator is parameter-dependent elliptic it induces isomorphisms

$$
A(\tau+i \gamma, \lambda): H^{s}(M) \rightarrow H^{s-\mu}(M)
$$

for all $s \in \mathbb{R}$ and all $|\tau, \lambda| \geq C$ for some constant $C>0$. The construction of [8] shows that for every $c^{\prime} \leq c^{\prime \prime}$ there exists a $C\left(c^{\prime}, c^{\prime \prime}\right)>0$ such that the operators (8) are isomorphisms for all $s \in \mathbb{R}$ and all $\left\{w \in \mathbb{C}: c^{\prime} \leq \operatorname{Im} w \leq c^{\prime \prime}\right\}$, provided that $|\lambda| \geq C\left(c^{\prime}, c^{\prime \prime}\right)$.

Also for boundary value problems we look at holomorphic families. A simplest example are parameter-dependent elliptic differential operators

$$
A(\tau, \lambda)=\sum_{|\alpha| \leq \mu} a_{\alpha}(x) D_{(x, \tau, \lambda)}^{\alpha}: H^{s}(\operatorname{int} X) \rightarrow H^{s-\mu}(\operatorname{int} X)
$$

in $X=\bar{G}$ for an open bounded set $G \subset \mathbb{R}^{n}$ with smooth boundary $Y$. In the parameter $(\tau, \lambda) \in \mathbb{R}^{1+l}$ we also admit $l=0$. Let

$$
T(\tau, \lambda)={ }^{\mathrm{t}}\left(T_{1}(\tau, \lambda), \ldots, T_{N}(\tau, \lambda)\right)
$$

be differential boundary conditions which are of the form

$$
T_{j}(\tau, \lambda) u=\left.\sum_{|\beta| \leq \mu_{j}} b_{\beta}(x) D_{(x, \tau, \lambda)}^{\beta} u\right|_{Y}
$$


with coefficients $b_{\beta}(x)$ that are smooth in a neighbourhood of $Y$. Then the family of operators

$$
\mathcal{A}(\tau, \lambda):=\left(\begin{array}{c}
A(\tau, \lambda) \\
T(\tau, \lambda)
\end{array}\right): H^{s}(\operatorname{int} X) \rightarrow \begin{gathered}
H^{s-\mu}(\operatorname{int} X) \\
\oplus_{j=1}^{N} H^{s-\mu_{j}-\frac{1}{2}}(Y)
\end{gathered}
$$

which is continuous for all $s>\max \left\{\mu_{j}+\frac{1}{2}: j=1, \ldots, N\right\}$ has a canonical extension to a family $\mathcal{A}(w, \lambda)$ which is holomorphic in $w \in \mathbb{C}$. Assuming that $\mathcal{A}(\tau, \lambda)$ is parameter-dependent elliptic it can easily be verified that $\mathcal{A}_{\gamma}(\tau, \lambda):=\mathcal{A}(\tau+i \gamma, \lambda)$ is also parameter-dependent elliptic for every $\gamma \in \mathbb{R}$. From Theorem 1.3 it follows that for every fixed $\gamma \in \mathbb{R}$ the operator family $\mathcal{A}_{\gamma}(\tau, \lambda)$ has a parameter-dependent parametrix $\mathcal{P}_{\gamma}(\tau, \lambda)$ of type $(d-\mu)^{+}$(and with upper left corner being of order $-\mu$ ).

Let us now assume $l=0$. Then $\mathcal{P}_{\gamma}(\tau)$ is known to be extendible to a (meromorphic) family $\mathcal{P}(w)$ of operators with a pattern of 'poles' of finite multiplicity which intersects every finite strip $\left\{w \in \mathbb{C}: c \leq \operatorname{Im} w \leq c^{\prime}\right\}$ in finitely many points. In order to analyse this behaviour in connection with the program of the papers Gohberg and Sigal [3] or of the discussion in [14], [4] we want to reduce orders and types of $\mathcal{P}(w)$ in such a way that the multiplicities of non-bijectivity points of $\mathcal{A}(w)$ (or of the poles of $\mathcal{P}(w)$ ) remain unchanged. For this reason we recall a result on holomorphic order reducing families also on a manifold with boundary.

Let $X$ be locally near a point of the boundary identified with the half-space $\overline{\mathbb{R}}_{+}^{n}=\{x \in$ $\left.\mathbb{R}^{n}: x_{n} \geq 0\right\}$; write $x=\left(x^{\prime}, x_{n}\right)$ and $\xi=\left(\xi^{\prime}, \xi_{n}\right)$. We then consider parameter-dependent symbols in $\mathbb{R}^{n}$ of the form

$$
r_{-}^{\mu}\left(\xi^{\prime}, \xi_{n}, \tau, \lambda\right):=\left(\varphi\left(\frac{\xi_{n}}{C\left\langle\xi^{\prime}, \tau, \lambda\right\rangle}\right)\left\langle\xi^{\prime}, \tau, \lambda\right\rangle-i \xi_{n}\right)^{\mu},
$$

$\mu \in \mathbb{Z}$, for a function $\varphi \in \mathcal{S}(\mathbb{R})$ such that $\varphi(0)=1$ and $\operatorname{supp} F^{-1} \varphi \subset \mathbb{R}_{+}$(with $F$ being the one-dimensional Fourier transform). As is known (10) is a classical symbol, cf. [11]. A sufficiently large choice of the constant $C>0$ guarantees the parameter-dependent ellipticity of (10). In order to form operators on $X$ we consider symbols

$$
\tilde{r}_{-}^{\mu}\left(x_{n}, \xi, \tau, \lambda\right):=r_{-}^{\mu}(\xi, \tau, \lambda)^{\omega\left(x_{n}\right)}\langle\xi, \tau, \lambda\rangle^{\mu\left(1-\omega\left(x_{n}\right)\right)}
$$

for some cut-off function $\omega\left(x_{n}\right)$. By a system of charts on $2 X$ to $\mathbb{R}^{n} \ni\left(x^{\prime}, x_{n}\right)$ for neighbourhoods of the boundary which induce charts on $Y$ to $\mathbb{R}^{n-1} \ni x^{\prime}$ and charts to $\mathbb{R}^{n}$ for neighbourhoods on $2 X \backslash Y$ we easily find a family

$$
\tilde{R}^{\mu}(\tau, \lambda) \in L_{\mathrm{cl}}^{\mu}\left(2 X ; \mathbb{R}_{\tau, \lambda}^{1+l}\right)_{\operatorname{tr}}
$$

of parameter-dependent elliptic pseudo-differential operators having the local amplitude functions (11) near $Y$ and $\langle\xi, \tau, \lambda\rangle^{\mu}$ outside a neighbourhood of $Y$, modulo lower order terms, cf. the paper [5]. More generally, a similar construction can be carried out for the case of operators acting in distributional sections of a vector bundle $\tilde{E} \in \operatorname{Vect}(2 X)$ and we then obtain $\tilde{R}^{\mu}(\tau, \lambda) \in L_{\mathrm{cl}}^{\mu}\left(2 X ; \tilde{E}, \tilde{E} ; \mathbb{R}_{\tau, \lambda}^{1+l}\right)_{\mathrm{tr}}$. Then

$$
\mathrm{r}^{+} \tilde{R}^{\mu}(\tau, \lambda) \mathrm{e}^{+}
$$

is an element in $\mathcal{B}^{\mu, 0}\left(X ; E, E ; \mathbb{R}^{1+l}\right)$ for $E=\left.\tilde{E}\right|_{X}$. We now apply the kernel cut-off construction from [13] to boundary value problems (which is also the background of the Theorems 1.5 and 1.6 below). Let us set

$$
K(\rho, \lambda):=(2 \pi)^{-1} \int_{-\infty}^{\infty} e^{i \rho \tau} \mathrm{r}^{+} \tilde{R}^{\mu}(\tau, \lambda) \mathrm{e}^{+} d \tau
$$


(the integration is carried out for the function in $\tau$ with values in the Fréchet space $\mathcal{B}^{\mu, 0}\left(X ; E, E ; \mathbb{R}^{l}\right)$ ). Moreover, choose an arbitrary function $\psi(\rho) \in C_{0}^{\infty}(\mathbb{R})$ which is equal to 1 in a neighbourhood of $\rho=0$, and form

$$
R^{\mu}(w, \lambda):=\int e^{-i w \rho} \psi(\rho) K(\rho, \lambda) d \rho .
$$

Then we obtain a family

$$
R^{\mu}(w, \lambda) \in \mathcal{A}\left(\mathbb{C}_{w}, \mathcal{B}^{\mu, 0}\left(X ; E, E ; \mathbb{R}^{l}\right)\right)
$$

which has the property

$$
\left.R^{\mu}(w, \lambda)\right|_{\operatorname{Im} w=0}=\mathrm{r}^{+} \tilde{R}^{\mu}(\tau, \lambda) \mathrm{e}^{+} \bmod \mathcal{B}^{-\infty, 0}\left(X ; E, E ; \mathbb{R}^{1+l}\right)
$$

(here $w=\tau+i \gamma)$.

Theorem 1.5 The operator family $R_{\gamma}^{\mu}(\tau, \lambda):=R^{\mu}(\tau+i \gamma, \lambda)$ is parameter-dependent elliptic in $\mathcal{B}^{\mu, 0}\left(X ; E, E ; \mathbb{R}^{1+l}\right)$ for every $\gamma \in \mathbb{R}$ (without additional trace and potential conditions), and for every $c^{\prime} \leq c^{\prime \prime}$ there exists a constant $C\left(c^{\prime}, c^{\prime \prime}\right)>0$ such that for $|\lambda| \geq C\left(c^{\prime}, c^{\prime \prime}\right)$ the operators

$$
R^{\mu}(w, \lambda): H^{s}(X, E) \rightarrow H^{s-\mu}(X, E)
$$

are isomorphisms for all $s \in \mathbb{R}, s>\max (\mu, 0)-\frac{1}{2}$, for all $\left\{w \in \mathbb{C}: c^{\prime} \leq \operatorname{Im} w \leq c^{\prime \prime}\right\}$.

This result corresponds to [5, Theorem 2.7].

Let us now describe a space of meromorphic families of pseudo-differential boundary value problems, first for the $\mathcal{B}^{\mu, d}$-version and then for $\mathcal{B}^{\mu, \mathrm{d}}$ in general.

Let $\mathcal{B}^{\mu, \mathrm{d}}\left(X ; \boldsymbol{v} ; \mathbb{C} \times \mathbb{R}^{l}\right)$ denote the subspace of all $h(w, \lambda) \in \mathcal{A}\left(\mathbb{C}, \mathcal{B}^{\mu, \mathrm{d}}\left(X ; \boldsymbol{v} ; \mathbb{R}^{l}\right)\right)$ such that $h(\tau+i \gamma, \lambda) \in \mathcal{B}^{\mu, \mathrm{d}}\left(X ; \boldsymbol{v} ; \mathbb{R}_{\tau, \lambda}^{1+l}\right)$ for every $\gamma \in \mathbb{R}$, uniformly in compact $\gamma$-intervals. If the upper left corner only contains a Green contribution, cf., analogously, the expression (5), we admit $\mu \in \mathbb{R}$ and also denote the corresponding space by $\mathcal{B}_{G}^{\mu, \mathrm{d}}\left(X ; \boldsymbol{v} ; \mathbb{C} \times \mathbb{R}^{l}\right)$. Otherwise, if the interior pseudo-differential part in the upper left corner is non-trivial, we assume $\mu \in \mathbb{Z}$. This allows us to assume arbitrary orders for all entries separately (and integer orders in the upper left corners) which gives us analogously the spaces $\mathcal{B}^{\boldsymbol{\mu}, \mathrm{d}}\left(X ; \boldsymbol{v} ; \mathbb{C} \times \mathbb{R}^{l}\right)$.

Let us now fix a sequence

$$
R=\left\{\left(p_{j}, m_{j}, G_{j}\right)\right\}_{j \in \mathbb{Z}}
$$

with $p_{j} \in \mathbb{C}, m_{j} \in \mathbb{N},\left|\operatorname{Im} p_{j}\right| \rightarrow \infty$ as $j \rightarrow \infty$, and finite-dimensional subspaces $G_{j} \subset$

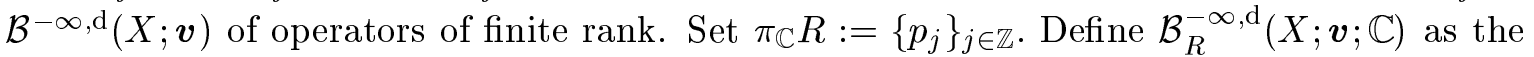
subspace of all $f(w) \in \mathcal{A}\left(\mathbb{C} \backslash \pi_{\mathbb{C}} R, \mathcal{B}^{-\infty, \mathrm{d}}(X ; \boldsymbol{v})\right)$ such that $f$ is meromorphic with poles at the points $p_{j}$ of multiplicities $m_{j}+1$ and Laurent coefficients at $\left(w-p_{j}\right)^{-(k+1)}$ in $G_{j}, 0 \leq k \leq m_{j}$, for all $j \in \mathbb{Z}$, and such that

$$
\chi(\tau+i \gamma) f(\tau+i \gamma) \in \mathcal{B}^{-\infty, \mathrm{d}}\left(X ; \boldsymbol{v} ; \mathbb{R}_{\tau}\right)
$$

for every $\gamma \in \mathbb{R}$, uniformly in compact $\gamma$-intervals. Here $\chi(w)$ is any $\pi_{\mathbb{C}} R$-excision function (i.e., $\chi \in C^{\infty}(\mathbb{C}), \chi \equiv 0$ for $\operatorname{dist}\left(w, \pi_{\mathbb{C}} R\right)<c_{0}, \chi \equiv 1$ for $\operatorname{dist}\left(w, \pi_{\mathbb{C}} R\right)>c_{1}$ for certain $\left.0<c_{0}<c_{1}\right)$. In a similar manner we can define spaces of the kind $\mathcal{B}_{R}^{-\infty, d}\left(X ; \boldsymbol{v} ; S_{\left(c^{\prime}, c^{\prime \prime}\right)}\right)$ of meromorphic functions in the strip $S_{\left(c^{\prime}, c^{\prime \prime}\right)}:=\left\{w: c^{\prime}<\operatorname{Im} w<c^{\prime \prime}\right\}$ for arbitrary $c^{\prime} \leq c^{\prime \prime}$, where $R$ is a sequence of the kind (12) with poles $p_{j} \in S_{\left(c^{\prime}, c^{\prime \prime}\right)}, \pi_{\mathbb{C}} R \cap S_{\left(c^{\prime}+\varepsilon, c^{\prime \prime}-\varepsilon\right)}$ finite for every $\varepsilon>0$, and corresponding multiplicities and Laurent coefficients as before.

We now set

$$
\mathcal{B}_{R}^{\mu, \mathrm{d}}\left(X ; \boldsymbol{v} ; S_{\left(c^{\prime}, c^{\prime \prime}\right)}\right):=\mathcal{B}^{\mu, \mathrm{d}}(X ; \boldsymbol{v} ; \mathbb{C})+\mathcal{B}_{R}^{-\infty, \mathrm{d}}\left(X ; \boldsymbol{v} ; S_{\left(c^{\prime}, c^{\prime \prime}\right)}\right) .
$$


in the sense of a non-direct sum. In particular, for $c^{\prime}=-\infty, c^{\prime \prime}=+\infty$ we have the space $\mathcal{B}_{R}^{\mu, \mathrm{d}}(X ; \boldsymbol{v} ; \mathbb{C})$.

Let us mainly discuss the latter case; all assertions have a corresponding version also in strips $S_{\left(c^{\prime}, c^{\prime \prime}\right)}$ for arbitrary $c^{\prime}, c^{\prime \prime}$.

Theorem 1.6 (i) For even $f(\tau) \in \mathcal{B}^{\boldsymbol{\mu}, \mathrm{d}}(X ; \boldsymbol{v} ; \mathbb{R})$ there exists an $h(w) \in \mathcal{B}^{\boldsymbol{\mu}, \mathrm{d}}(X ; \boldsymbol{v} ; \mathbb{C})$ such that

$$
\left.h(w)\right|_{\operatorname{Im} w=0}=f(\tau) \bmod \mathcal{B}^{-\infty, \mathrm{d}}(X ; \boldsymbol{v} ; \mathbb{R}) .
$$

(ii) If $f(\tau)$ is parameter-dependent elliptic, also $h(\tau+i \gamma) \in \mathcal{B}^{\boldsymbol{\mu}, \mathrm{d}}\left(X ; \boldsymbol{v} ; \mathbb{R}_{\tau}\right)$ is parameterdependent elliptic for every $\gamma \in \mathbb{R}$.

Proof. Cf. [6, Theorem 2.1.68].

Definition 1.7 Let $R$ be a sequence of the form (12). Then an element $\mathcal{B}_{R}^{\boldsymbol{\mu}, \mathrm{d}}\left(X ; \boldsymbol{v} ; S_{\left(c^{\prime}, c^{\prime \prime}\right)}\right)$ is called elliptic if for some $\gamma \in \mathbb{R}, c^{\prime}<\gamma<c^{\prime \prime}$, such that $\{\operatorname{Im} w=\gamma\} \cap \pi_{\mathbb{C}} R=\varnothing$ the family $f(\tau+i \gamma) \in \mathcal{B}^{\boldsymbol{\mu}, \mathrm{d}}\left(X ; \boldsymbol{v} ; \mathbb{R}_{\tau}\right)$ is parameter-dependent elliptic, cf. Definition 1.1.

Remark 1.8 The ellipticity of $f$ in the sense of Definition 1.7 is independent of the specific choice of $\gamma$.

The following result may be found in [12] (except for the types in the inverse which are calculated in [6]); concerning more material see also [8].

Theorem 1.9 Let $f \in \mathcal{B}_{R}^{\mu, \mathrm{d}}\left(X ; \boldsymbol{v} ; S_{\left(c^{\prime}, c^{\prime \prime}\right)}\right)$ be elliptic. Then there exists an inverse $f^{-1} \in$ $\mathcal{B}_{Q}^{-\mu,(\mathrm{d}-\mu)^{+}}\left(X ; \boldsymbol{v}^{-1} ; S_{\left(c^{\prime}, c^{\prime \prime}\right)}\right)$ (in the corresponding space of meromorphic operator functions) for a certain sequence $Q$ of analogous structure as $R$. Here $\boldsymbol{v}^{-1}:=(F, E ; G, J)$.

Proof. From [6, Theorem 1.2.92] we know that $f(\tau+i \gamma)$ has a parametrix $f^{(-1)}(\tau+i \gamma)$ in the space $\mathcal{B}^{-\mu,(\mu-d)^{+}}\left(X ; \boldsymbol{v}^{-1} ; \mathbb{R}_{\tau}\right)$ for any fixed $\gamma \in\left(c^{\prime}, c^{\prime \prime}\right)$. From Theorem 1.6 it follows the existence of a $g(w) \in \mathcal{B}^{-\mu,(d-\mu)^{+}}\left(X ; \boldsymbol{v}^{-1} ; \mathbb{C}\right)$ such that $f^{(-1)}(\tau+i \gamma)=g(\tau+i \gamma)$ $\bmod \mathcal{B}^{-\infty,(d-\mu)^{+}}\left(X ; \boldsymbol{v}^{-1} ; \mathbb{R}\right)$. This implies $f(w) g(w)=1-c(w)$ for some $c(w) \in \mathcal{B}_{S}^{-\infty,(d-\mu)^{+}}\left(X ; \boldsymbol{v}_{r} ; S_{\left(c^{\prime}, c^{\prime \prime}\right)}\right)$ for some sequence $S$ of the kind $(12), \boldsymbol{v}_{r}=(F, F ; G, G)$. As is shown in [12] we have $(1-c)^{-1}=(1-d)$ for an element $d \in \mathcal{B}_{T}^{-\infty,(d-\mu)^{+}}\left(X ; \boldsymbol{v}_{r} ; S_{\left(c^{\prime}, c^{\prime \prime}\right)}\right)$. This gives us the inverse $f^{-1}(w)=f^{(-1)}(w)(1-d(w))$ as an element in $\mathcal{B}^{-\mu,(d-\mu)^{+}}\left(X ; \boldsymbol{v}^{-1} ; S_{\left(c^{\prime}, c^{\prime \prime}\right)}\right)$.

Remark 1.10 The operator families $R^{\mu}(w, \lambda)$ of Theorem 1.5 belong to $\mathcal{B}^{\mu, 0}\left(X ; E, E ; \mathbb{C} \times \mathbb{R}^{l}\right)$. According to Theorem 1.9 for every fixed $\lambda \in \mathbb{R}$ we obtain $\left(R^{\mu}(w, \lambda)\right)^{-1} \in \mathcal{B}_{R}^{-\mu, 0}(X ; E, E ; \mathbb{C})$ for some sequence $R=R(\lambda)$ as described in (12). In particular, for every $c^{\prime} \leq c^{\prime \prime}$ and sufficiently large $|\lambda|$ we obtain $\pi_{\mathbb{C}} R(\lambda) \cap S_{\left(c^{\prime}, c^{\prime \prime}\right)}=\varnothing$. Similar constructions are possible on closed $C^{\infty}$ manifolds $M$.

Remark 1.11 Let $f \in \mathcal{B}^{\boldsymbol{\mu}, \mathrm{d}}\left(X ; \boldsymbol{v} ; S_{\left(c^{\prime}, c^{\prime \prime}\right)}\right)$ be elliptic. Then $f$ defines a meromorphic Fredholm family between the spaces as in formula (1) for all $s>\max (\mu, \mathrm{d})-\frac{1}{2}$, and $f^{-1}$ is of analogous nature. Thus we are in the context of the paper [3] of Gohberg and Sigal, and we can apply the definition of partial null-multiplicities $\boldsymbol{n}\left(f\left(w_{0}\right)\right)$ and partial polar multiplicities $\boldsymbol{p}\left(f\left(w_{0}\right)\right):=\boldsymbol{n}\left(f^{-1}\left(w_{0}\right)\right)$ at every $w_{0} \in S_{\left(c^{\prime}, c^{\prime \prime}\right)}$. There are only finitely many points $w_{0} \in S_{\left(c^{\prime}+\varepsilon, c^{\prime \prime}-\varepsilon\right)}$ for every $\varepsilon>0$, where $\boldsymbol{n}\left(f\left(w_{0}\right)\right)$ and $\boldsymbol{p}\left(f\left(w_{0}\right)\right)$ do not vanish. Thus, the numbers

$$
\boldsymbol{n}_{\varepsilon}(f):=\sum_{\operatorname{Im} w_{0} \in\left(c^{\prime}+\varepsilon, c^{\prime \prime}-\varepsilon\right)} \boldsymbol{n}\left(f\left(w_{0}\right)\right), \boldsymbol{p}_{\varepsilon}(f):=\sum_{\operatorname{Im} w_{0} \in\left(c^{\prime}+\varepsilon, c^{\prime \prime}-\varepsilon\right)} \boldsymbol{p}\left(f\left(w_{0}\right)\right)
$$

are both finite. 
For the constructions in Chapter 2 below it will be convenient to reduce orders and types to zero in such a way that we do not destroy poles and multiplicities in a prescribed strip $S_{\left(c^{\prime}, c^{\prime \prime}\right)}$. From Remark 1.10 for every $c^{\prime} \leq c^{\prime \prime}$ we have elliptic families

$$
R_{E}^{\mu}(w): H^{s}(X, E) \rightarrow H^{s-\mu}(X, E)
$$

in $\mathcal{B}^{\mu, 0}(X ; E, E ; \mathbb{C}), \mu \in \mathbb{Z}, s>\max (\mu, 0)-\frac{1}{2}$, and

$$
P_{J}^{\nu}(w): H^{s}(Y, J) \rightarrow H^{s-\nu}(Y, J)
$$

in $L_{\mathrm{cl}}^{\nu}(Y ; J, J ; \mathbb{C}), \nu \in \mathbb{R}, s \in \mathbb{R}$, such that the families of inverses are holomorphic in the strip $S_{\left(c^{\prime}, c^{\prime \prime}\right)}$ (outside $S_{\left(c^{\prime}, c^{\prime \prime}\right)}$ there may be poles as described in Theorem 1.9).

Consider now operator families $(1)$ of the class $\mathcal{B}_{R}^{\boldsymbol{\mu}, \boldsymbol{d}}(X ; \boldsymbol{v} ; \mathbb{C})$ for a certain (12). Let $s \in \mathbb{N}, s>\max (\mu, d)-\frac{1}{2}$, and set

$$
\begin{gathered}
\mathcal{R}_{1}(w):=\operatorname{diag}\left(R_{E}^{s}(w),\left(P_{J_{k}}^{s-\frac{1}{2}-\nu_{k}}(w)\right)_{k=1, \ldots, K}\right), \\
\mathcal{R}_{2}(w):=\operatorname{diag}\left(R_{F}^{s-\mu}(w),\left(P_{G_{j}}^{s-\frac{1}{2}-\mu_{j}}(w)\right)_{j=1, \ldots, J}\right) .
\end{gathered}
$$

Then the composition

$$
\mathcal{A}_{0}(w):=\mathcal{R}_{2}(w) \mathcal{A}(w) \mathcal{R}_{1}^{-1}(w)
$$

induces an isomorphism

$$
\mathcal{B}_{R}^{\boldsymbol{\mu}, d}\left(X ; \boldsymbol{v} ; S_{\left(c^{\prime}, c^{\prime \prime}\right)}\right) \rightarrow \mathcal{B}_{Q}^{0,0}\left(X ; \boldsymbol{v} ; S_{\left(c^{\prime}, c^{\prime \prime}\right)}\right) ; \mathcal{A}(w) \rightarrow \mathcal{A}_{0}(w),
$$

for every fixed choice of $c^{\prime} \leq c^{\prime \prime}$, provided that the absolute value of the hidden parameter $\lambda$ in the operators $\mathcal{R}_{j}(w), j=1,2$, is chosen large enough. Moreover, the partial null- and polar indices of the functions $\mathcal{A}(w)$ and $\mathcal{A}_{0}(w)$ coincide. Here $Q$ is a resulting sequence of the kind (12).

\section{Calculus on infinite Cylinders}

\subsection{Operators in weighted Sobolev spaces}

Let $M$ be a closed compact $C^{\infty}$ manifold, and let $E \in \operatorname{Vect}(M)$. Moreover, let $P_{E}^{\mu}(\tau) \in$ $L_{\mathrm{cl}}^{\mu}\left(M ; E, E ; \mathbb{R}_{\tau}\right)$ be an arbitrary parameter-dependent elliptic operator which induces isomorphisms $P_{E}^{\mu}(\tau): H^{s}(M, E) \rightarrow H^{s-\mu}(M, E)$ for all $s, \tau \in \mathbb{R}$. Then $H^{s}(\mathbb{R} \times M, E)$ for $s \in \mathbb{R}$ is defined as the completion of $C_{0}^{\infty}(\mathbb{R} \times M, E)$ with respect to the norm

$$
\left\{\int_{\mathbb{R}}\left\|P_{E}^{s}(\tau) F u(\tau)\right\|_{L^{2}(M, E)}^{2} d \tau\right\}^{\frac{1}{2}},
$$

where $F$ is the Fourier transform. (For convenience $E \in \operatorname{Vect}(\mathbb{R} \times M)$ is identified with the pull back of the former $E \in \operatorname{Vect}(M)$ to the cylinder under der canonical projection $\mathbb{R} \times M \rightarrow M)$. Clearly the space $H^{s}(\mathbb{R} \times M, E)$ is independent of the specific choice of the order reducing family. Moreover, for every $\gamma \in \mathbb{R}$ we set

$$
H^{s, \gamma}(\mathbb{R} \times M, E):=e^{-t \gamma} H^{s}(\mathbb{R} \times M, E) .
$$

For the case of a compact $C^{\infty}$ manifold $X$ with boundary $Y$ we set

$$
H^{s, \gamma}(\mathbb{R} \times \operatorname{int} X, E):=\left.H^{s, \gamma}(\mathbb{R} \times 2 X, \tilde{E})\right|_{\mathbb{R} \times \operatorname{int} X}
$$


for some $\tilde{E} \in \operatorname{Vect}(\mathbb{R} \times 2 X)$ such that $E=\left.\tilde{E}\right|_{\mathbb{R} \times X}$. Here $2 X$ is same as in Section 1.1. The norm in (17) is defined by means of an isomorphism to $H^{s, \gamma}(\mathbb{R} \times 2 X, \tilde{E}) / H_{0}^{s, \gamma}\left(\mathbb{R} \times \operatorname{int} X_{-}, \tilde{E}\right)$ where $H_{0}^{s, \gamma}\left(\mathbb{R} \times \operatorname{int} X_{-}, \tilde{E}\right)$ means the (closed) subspace of all $u \in H^{s, \gamma}(\mathbb{R} \times 2 X, \tilde{E})$ such that $\left.u\right|_{\mathbb{R} \times \operatorname{int} X}$ vanishes.

For abbreviation we now set

$\mathcal{H}^{s, \gamma}(\mathbb{R} \times X ; E, J):=H^{s, \gamma}(\mathbb{R} \times \operatorname{int} X, E) \oplus H^{s, \gamma}(\mathbb{R} \times Y, J), \quad E \in \operatorname{Vect}(\mathbb{R} \times X), J \in \operatorname{Vect}(\mathbb{R} \times Y)$, $s, \gamma \in \mathbb{R}$.

Let $f \in \mathcal{B}_{R}^{0,0}\left(X ; \boldsymbol{v} ; S_{\left(c^{\prime}, c^{\prime \prime}\right)}\right)$ and set

$$
\mathrm{op}^{\gamma}(f) u(t):=(2 \pi)^{-1} \int_{I_{\gamma}} e^{i t w} f(w) F u(w) d w,
$$

where $I_{\gamma}:=\{w \in \mathbb{C}: w=\tau+i \gamma, \tau \in \mathbb{R}\}, \gamma \in\left(c^{\prime}, c^{\prime \prime}\right)$, and $I_{\gamma} \cap \pi_{\mathbb{C}} R=\varnothing$. In (18) we first take $u \in C_{0}^{\infty}(\mathbb{R} \times X, E) \oplus C_{0}^{\infty}(\mathbb{R} \times Y, J)$; then $(F u)(w)$ is an entire function in $w$.

Proposition 2.1 (i) The operator $C_{0}^{\infty}(\mathbb{R} \times X, E) \oplus C_{0}^{\infty}(\mathbb{R} \times Y, J) \rightarrow C^{\infty}(\mathbb{R} \times X, F) \oplus$ $C^{\infty}(\mathbb{R} \times Y, G)$ given by (18) extends to a continuous map

$$
\mathrm{op}^{\gamma}(f): \mathcal{H}^{s, \gamma}(\mathbb{R} \times X ; E, J) \rightarrow \mathcal{H}^{s, \gamma}(\mathbb{R} \times X ; F, G)
$$

for every $s>-\frac{1}{2}$.

(ii) If $f(w)$ is bijective for all $w \in I_{\gamma}$, then (19) is an isomorphism for every $s>-\frac{1}{2}$.

Proof. (i) First observe that the space $\mathcal{H}^{s, \gamma}(\mathbb{R} \times X ; E, J)$ can equivalently be defined as the completion of $C_{0}^{\infty}\left(\mathbb{R}, C^{\infty}(X, E) \oplus C^{\infty}(Y, J)\right)$ with respect to the norm

$$
\left\{\int_{I_{\gamma}}\left\|\mathcal{R}_{E, J}^{s}(\tau) F u(\tau)\right\|_{H^{0}(X, E) \oplus H^{0}(Y, J)}^{2} d \tau\right\}^{\frac{1}{2}}
$$

when we set $\mathcal{R}_{E, J}^{s}(\tau):=\operatorname{diag}\left(R_{E}^{s}(\tau), P_{J}^{s}(\tau)\right)$ with the abovementioned order reducing families $R_{E}^{s}(\tau)$ and $P_{J}^{s}(\tau)$, respectively. Then the proof of (i) is formally analogous to the corresponding result in the case without boundary, cf. [14].

(ii) If $f(w)$ is bijective on the line $I_{\gamma}$ from the proof of Theorem 1.9 we know that $f^{-1}(\tau+$ $i \gamma$ ) belongs to $\mathcal{B}^{0,0}\left(X ; \boldsymbol{v}^{-1} ; \mathbb{R}_{\tau}\right)$. The bijectivity of $\mathrm{op}^{\gamma}(f)$ then follows from $\left(\mathrm{op}^{\gamma}(f)\right)^{-1}=$ $\mathrm{op}^{\gamma}\left(f^{-1}\right)$, using the fact that $\mathrm{op}^{\gamma}(f) \mathrm{op}^{\gamma}(g)=\mathrm{op}^{\gamma}(f g)$ for arbitrary operator-valued amplitude functions $f$ and $g$ in our spaces.

We now introduce spaces with double weights $\boldsymbol{\delta}:=\left(\delta_{-}, \delta_{+}\right), \delta_{ \pm} \in \mathbb{R}$, as

$$
\mathcal{H}^{s, \boldsymbol{\delta}}(\mathbb{R} \times X ; E, J):=\sigma(t) \mathcal{H}^{s, \delta_{-}}(\mathbb{R} \times X ; E, J)+(1-\sigma(t)) \mathcal{H}^{s, \delta_{+}}(\mathbb{R} \times X ; E, J)
$$

in the sense of a non-direct sum for any $\sigma \in C^{\infty}(\mathbb{R})$ such that $\sigma(t)=1$ for $t<c_{0}$ and $\sigma(t)=0$ for $t>c_{1}$ for certain $c_{0}<c_{1}$. A norm on the space $\mathcal{H}^{s, \delta}(\mathbb{R} \times X ; E, J)$ is defined by

$$
\|u\|_{\mathcal{H}^{s, \delta}(\mathbb{R} \times X ; E, J)}^{2}=\|\sigma u\|_{\mathcal{H}^{s, \delta}-(\mathbb{R} \times X ; E, J)}^{2}+\|(1-\sigma) u\|_{\mathcal{H}^{s, \delta}+(\mathbb{R} \times X ; E, J)}^{2} ;
$$

of course, the specific choice of $\sigma$ is unimportant. 


\subsection{Relative index}

Lemma 2.2 For $\delta_{-} \leq \delta_{+}$we have $u \in \mathcal{H}^{s, \boldsymbol{\delta}}(\mathbb{R} \times X ; E, J)$ if and only if $u \in \mathcal{H}^{s, \gamma}(\mathbb{R} \times X ; E, J)$ for every $\gamma \in\left[\delta_{-}, \delta_{+}\right]$.

Proof. Let $u \in \mathcal{H}^{s, \boldsymbol{\delta}}(\mathbb{R} \times X ; E, J)$. For any $\gamma \in\left[\delta_{-}, \delta_{+}\right]$we have

$$
\begin{aligned}
\|u\|_{\mathcal{H}^{s, \gamma}(\mathbb{R} \times X ; E, J)}^{2} & \leq 2\left(\|\sigma u\|_{\mathcal{H}^{s, \gamma}(\mathbb{R} \times X ; E, J)}^{2}+\|(1-\sigma) u\|_{\mathcal{H}^{s, \gamma}(\mathbb{R} \times X ; E, J)}^{2}\right) \\
& \leq c\|u\|_{\mathcal{H}^{s, \delta}(\mathbb{R} \times X ; E, J)}^{2},
\end{aligned}
$$

since

$$
\|\sigma u\|_{\mathcal{H}^{s, \gamma}(\mathbb{R} \times X ; E, J)}^{2} \leq c_{1}\|\sigma u\|_{\mathcal{H}^{s, \delta}-(\mathbb{R} \times X ; E, J)}^{2}
$$

and

$$
\|(1-\sigma) u\|_{\mathcal{H}^{s, \gamma}(\mathbb{R} \times X ; E, J)}^{2} \leq c_{1}\|(1-\sigma) u\|_{\mathcal{H}^{s, \delta_{+}}(\mathbb{R} \times X ; E, J)}^{2} .
$$

Here the constants $c_{1}, c_{2}$ and hence $c$ are independent of $u$.

Conversely, let $u \in \mathcal{H}^{s, \gamma}(\mathbb{R} \times X ; E, J)$ for every $\gamma \in\left[\delta_{-}, \delta_{+}\right]$. The relation (20) shows that

$$
\|u\|_{\mathcal{H}^{s, \delta}(\mathbb{R} \times X ; E, J)}^{2} \leq c\left(\|u\|_{\mathcal{H}^{s, \delta}-(\mathbb{R} \times X ; E, J)}^{2}+\|u\|_{\mathcal{H}^{s, \delta_{+}(\mathbb{R} \times X ; E, J)}}^{2}\right),
$$

for the constant $c$ depending only on $\sigma$. The inequality (21) shows that when $u$ belongs both to $\mathcal{H}^{s, \delta_{-}}(\mathbb{R} \times X ; E, J)$ and $\mathcal{H}^{s, \delta_{+}}(\mathbb{R} \times X ; E, J)$ it follows that $u \in \mathcal{H}^{s, \delta}(\mathbb{R} \times X ; E, J)$.

Remark 2.3 If $u \in \mathcal{H}^{s, \boldsymbol{\delta}}(\mathbb{R} \times X ; E, J)$ it follows that $F u(w)$ is holomorphic in the strip $S_{\left(\delta_{-}, \delta_{+}\right)}$. This allows us to apply (18) for every $\delta_{-} \leq \gamma \leq \delta_{+}$such that $I_{\gamma} \cap \pi_{\mathbb{C}} R=\varnothing$.

Indeed, this follows from the definition of the weighted Sobolev spaces, i.e., elements $u$ contain the factors $e^{-\delta_{-} t}$ and $e^{-\delta_{+} t}$ in a neighbourhoods of $-\infty$ and $+\infty$, respectively.

Proposition 2.4 Let $f(w) \in \mathcal{B}_{R}^{0,0}\left(X ; \boldsymbol{v} ; S_{\left(c^{\prime}, c^{\prime \prime}\right)}\right)$ and let $c^{\prime}<\delta_{-} \leq \delta_{+}<c^{\prime \prime}, \pi_{\mathbb{C}} R \cap\left(I_{\delta_{-}} \cup\right.$ $\left.I_{\delta_{+}}\right)=\varnothing$. Then for every $u \in \mathcal{H}^{s, \boldsymbol{\delta}}(\mathbb{R} \times X ; E, J)$ we have

$$
\mathrm{op}^{\delta_{-}}(f) u(t)-\mathrm{op}^{\delta_{+}}(f) u(t)=2 \pi i \sum_{p \in S_{\left(\delta_{-}, \delta_{+}\right)}} \operatorname{res}_{p} e^{i t w} f(w) F u(w)
$$

Proof. According to the relation (13) the function $f$ has a representation $f(w)=f_{0}(w)+$ $f_{1}(w)$ for certain $f_{0}(w) \in \mathcal{B}^{0,0}(X ; \boldsymbol{v} ; \mathbb{C})$ and $f_{1}(w) \in \mathcal{B}_{R}^{-\infty, 0}\left(X ; \boldsymbol{v} ; S_{\left(c^{\prime}, c^{\prime \prime}\right)}\right)$. We first show that

$$
\mathrm{op}^{\delta_{-}}\left(f_{1}\right) u(t)-\mathrm{op}^{\delta_{+}}\left(f_{1}\right) u(t)=2 \pi i \sum_{p \in S_{\left(\delta_{-}, \delta_{+}\right)}} \operatorname{res}_{p} e^{i t w} f_{1}(w) F u(w)
$$

$\left(f_{1}(w)\right.$ on the right hand side may be replaced by $\left.f(w)\right)$ which is a consequence of Cauchy's integral formula and the Residue Theorem. Furthermore, for any $u \in C_{0}^{\infty}(\mathbb{R} \times X ; E) \oplus$ $C_{0}^{\infty}(\mathbb{R} \times Y ; J)$ by Cauchy's Theorem we obtain

$$
\mathrm{op}^{\delta_{-}}\left(f_{0}\right) u(t)=\mathrm{op}^{\delta_{+}}\left(f_{0}\right) u(t) .
$$

Since $C_{0}^{\infty}(\mathbb{R} \times X ; E) \oplus C_{0}^{\infty}(\mathbb{R} \times Y, J)$ is dense in $\mathcal{H}^{s, \gamma}(\mathbb{R} \times X ; E, J)$ for any $\gamma \in \mathbb{R}$, using Proposition 2.1 and Lemma 2.2 we get (22) for any $u \in \mathcal{H}^{s, \boldsymbol{\delta}}(\mathbb{R} \times X ; E, J)$. 
Our next objective is to associate with $f \in \mathcal{B}_{R}^{0,0}\left(X ; \boldsymbol{v} ; S_{\left(c^{\prime}, c^{\prime \prime}\right)}\right)$ a continuous operator

$$
\mathcal{A}: \mathcal{H}^{s, \boldsymbol{\delta}}(\mathbb{R} \times X ; E, J) \rightarrow \mathcal{H}^{s, \boldsymbol{\delta}}(\mathbb{R} \times X ; F, G)
$$

in the case $\delta_{-} \leq \delta_{+}$. Here and in the sequel we may first assume $s=0$; the conclusions then hold for arbitrary reals $s>-\frac{1}{2}$.

Let us set

$$
D(f):=\left\{u \in \mathcal{H}^{s, \boldsymbol{\delta}}(\mathbb{R} \times X ; E, J): \operatorname{res}_{p} e^{i t w} f(w) F u(w)=0 \text { for } p \in S_{\left(\delta_{-}, \delta_{+}\right)}\right\} .
$$

Thus op $\left.{ }^{\gamma}(f)\right|_{D(f)}$ is independent of the choice of $\gamma$ (and also of $s$ ).

Lemma 2.5 Let $f(w)$ be elliptic. Then $D(f)$ is a closed subspace of $\mathcal{H}^{s, \boldsymbol{\delta}}(\mathbb{R} \times X ; E, J)$ of finite codimension

$$
\operatorname{codim} D(f)=\sum_{p \in S_{\left(\delta_{-}, \delta_{+}\right)}} \boldsymbol{p}(f(p))
$$

(which is independent of $\gamma$ and $s$ ).

Proof. The proof is formally analogous to Lemma 3.8 in Harutjunjan and Schulze [4] or Lemma 6.5 in Schulze and Tarkhanov [14].

Proposition 2.6 $\quad$ (i) Let $f(w) \in \mathcal{B}_{R}^{0,0}\left(X ; \boldsymbol{v} ; S_{\left(c^{\prime}, c^{\prime \prime}\right)}\right)$ be elliptic and assume $\pi_{\mathbb{C}} R \cap I_{\delta_{ \pm}}=\varnothing$. Then $\mathrm{op}^{\gamma}(f)$ induces a continuous operator

$$
D(f) \rightarrow \mathcal{H}^{s, \boldsymbol{\delta}}(\mathbb{R} \times X ; F, G)
$$

for every $s>-\frac{1}{2}$.

(ii) Let $f(w)$ have no non-bijectivity points on the lines $I_{\delta_{ \pm}}$. Then for every $v \in \mathcal{H}^{s, \boldsymbol{\delta}}(\mathbb{R} \times$ $X ; F, G)$ there exists a solution $u \in D(f)$ of the equation $\mathrm{op}^{\gamma}(f) u=v$ if and only if

$$
\operatorname{res}_{p} e^{i t w} f^{-1}(w) F v(w)=0 \text { for } p \in S_{\left(\delta_{-}, \delta_{+}\right)}
$$

(by $R(f)$ we denote the set of all such functions $v \in \mathcal{H}^{s, \boldsymbol{\delta}}(\mathbb{R} \times X ; F, G)$ ).

Proof. (i) Because of Proposition 2.4 for $u \in D(f)$ we have

$$
\mathrm{op}^{\gamma}(f) u(t)=(2 \pi)^{-1} \int_{I_{\delta_{-}}} e^{i t w} f(w) F u(w) d w=(2 \pi)^{-1} \int_{I_{\delta_{+}}} e^{i t w} f(w) F u(w) d w .
$$

Now Proposition 2.1 (i) gives us that $\operatorname{op}^{\gamma}(f) u \in \mathcal{H}^{s, \delta_{ \pm}}(\mathbb{R} \times X ; F, G)$ and the proof follows from Lemma 2.2 .

(ii) Let $v=\mathrm{op}^{\gamma}(f) u$ for some $u \in D(f)$. Then $F v(w)=f(w) F u(w)$ or $f^{-1}(w) F v(w)=$ $F u(w)$. Since $F u(w)$ is a holomorphic function in the strip $S_{\left(\delta_{-}, \delta_{+}\right)}$we obtain $v \in R(f)$.

Conversely, let $v \in R(f)$. Then it is easy to show that the function $u$ defined as an integral

$$
u(t)=\frac{1}{2 \pi} \int_{I_{\gamma}} e^{i t w} f^{-1}(w) F v(w) d w
$$

which is independent of $\gamma \in\left[\delta_{-}, \delta_{+}\right]$, cf. Proposition 2.4, belongs to $D(f)$ and op ${ }^{\gamma}(f) u=$ $v$. 
Corollary 2.7 The operator (24) is injective, and $R(f)$ is a closed subspace of $\mathcal{H}^{s, \boldsymbol{\delta}}(\mathbb{R} \times$ $X ; F, G)$ of finite codimension

$$
\operatorname{codim} R(f)=\sum_{p \in S_{\left(\delta_{-}, \delta_{+}\right)}} \boldsymbol{n}(f(p))
$$

This is a consequence of Proposition 2.6 (ii) and Lemma 2.5 .

Let us now define the operator (23) by

$$
\mathcal{A} u=\mathrm{op}^{\gamma}(f) u \text { for } u \in D(f) \text { and } \mathcal{A} u=0 \text { for } u \in \mathcal{H}^{s, \boldsymbol{\delta}}(\mathbb{R} \times X ; E, J) \ominus D(f)
$$

(the latter orthogonal complement refers to the scalar product of $\mathcal{H}^{0, \boldsymbol{\delta}}(\mathbb{R} \times X ; E, J)$ ). Summing up, Lemma 2.5 and Corollary 2.7 give the following theorem.

Theorem 2.8 Let $f(w)$ have no non-bijectivity points on the lines $I_{\delta_{+}}, \delta_{-} \leq \delta_{+}$. Then the operator (23) defined by (25) is a Fredholm operator with

$$
\text { ind } \mathcal{A}=\sum_{p \in S_{\left(\delta_{-}, \delta_{+}\right)}}\{\boldsymbol{p}(f(p))-\boldsymbol{n}(f(p))\} .
$$

Next we associate operators $(23)$ with elements $f(w) \in \mathcal{B}_{R}^{0,0}\left(X ; \boldsymbol{v} ; S_{\left(c^{\prime}, c^{\prime \prime}\right)}\right)$ for the case $\delta_{-}>\delta_{+}$. To this end we pass to the formal transposed operators with respect to the bilinear pairings

$$
\mathcal{H}^{0, \boldsymbol{\delta}}(\mathbb{R} \times X ; E, J) \times \mathcal{H}^{0,-\boldsymbol{\delta}}\left(\mathbb{R} \times X ; E^{\prime}, J^{\prime}\right) \rightarrow \mathbb{C}
$$

induced by $\langle u, v\rangle:=(u, \bar{v})$ with the $\mathcal{H}^{0,(0,0)}(\mathbb{R} \times X ; E, J)$-scalar product $(\cdot, \cdot)$; here $E^{\prime}$ and $J^{\prime}$ are the dual bundles of $E$ and $J$, respectively. The formal adjoint of $\mathrm{op}^{\gamma}(f)$ is equal to $\mathrm{op}^{-\gamma}\left(f^{\prime}\right)$ for $f^{\prime}(w):={ }^{\mathrm{t}} f(-w)$ where the latter t means the pointwise transposed of $f$ which gives us an element in $\mathcal{B}_{R^{\prime}}^{0,0}\left(X ; \boldsymbol{v}^{\prime} ; S_{\left(-c^{\prime \prime},-c^{\prime}\right)}\right)$ for some resulting $R^{\prime}$, and $\boldsymbol{v}^{\prime}:=\left(F^{\prime}, E^{\prime} ; G^{\prime}, J^{\prime}\right)$. For ${ }^{\mathrm{t}} f(-w)$ we are now in the situation with weights $\delta_{-}^{\prime}:=-\delta_{-}<-\delta_{+}=: \delta_{+}^{\prime}$ as before, and hence there is a corresponding operator called ${ }^{\mathrm{t}} \mathcal{A}$, which is continuous in the sense

$$
{ }^{\mathrm{t}} \mathcal{A}: \mathcal{H}^{s, \boldsymbol{\delta}^{\prime}}\left(\mathbb{R} \times X ; F^{\prime}, G^{\prime}\right) \rightarrow \mathcal{H}^{s, \boldsymbol{\delta}^{\prime}}\left(\mathbb{R} \times X ; E^{\prime}, J^{\prime}\right),
$$

for $\boldsymbol{\delta}^{\prime}=\left(\delta_{-}^{\prime}, \delta_{+}^{\prime}\right), s>-\frac{1}{2}$ (first for $s=0$, but since we have operators in Boutet de Monvel's calculus we obtain continuity for all $s>-\frac{1}{2}$ ). Analogously we define

$$
D\left(f^{\prime}\right):=\left\{v \in \mathcal{H}^{s, \boldsymbol{\delta}^{\prime}}\left(\mathbb{R} \times X ; F^{\prime}, G^{\prime}\right): \operatorname{res}_{p} e^{i t w} f^{\prime}(w) F v(w)=0 \text { for } p \in S_{\left(\delta_{-}^{\prime}, \delta_{+}^{\prime}\right)}\right\}
$$

that is a closed subspace of $\mathcal{H}^{s, \boldsymbol{\delta}^{\prime}}\left(\mathbb{R} \times X ; F^{\prime}, G^{\prime}\right)$ of finite codimension

$$
\operatorname{codim} D\left(f^{\prime}\right)=\sum_{p \in S_{\left(\delta_{-}^{\prime}, \delta_{+}^{\prime}\right)}} \boldsymbol{p}\left(f^{\prime}(w)\right)=\sum_{p \in S_{\left(\delta_{+}, \delta_{-}\right)}} \boldsymbol{p}(f(w))
$$

Corollary 2.7 shows that if $f(w)$ has no non-bijectivity points on the lines $I_{\delta_{ \pm}}$the analogue of the operator $(24)$

$$
D\left(f^{\prime}\right) \rightarrow \mathcal{H}^{s, \boldsymbol{\delta}^{\prime}}\left(\mathbb{R} \times X ; E^{\prime}, J^{\prime}\right)
$$

is injective, and $R\left(f^{\prime}\right)$ is a closed subspace of $\mathcal{H}^{s, \boldsymbol{\delta}^{\prime}}\left(\mathbb{R} \times X ; E^{\prime}, J^{\prime}\right)$ of finite codimension

$$
\operatorname{codim} R\left(f^{\prime}\right)=\sum_{p \in S_{\left(\delta_{-}^{\prime}, \delta_{+}^{\prime}\right)}} \boldsymbol{n}\left(f^{\prime}(p)\right)=\sum_{p \in S_{\left(\delta_{+}, \delta_{-}\right)}} \boldsymbol{n}(f(p)) .
$$


Now using the fact that for every $u \in \mathcal{H}^{s, \boldsymbol{\delta}}(\mathbb{R} \times X ; E, J)$ there is a unique $f \in \mathcal{H}^{s, \boldsymbol{\delta}}(\mathbb{R} \times$ $X ; F, G)$ such that

$$
\langle v, f\rangle=\left\langle{ }^{\mathrm{t}} \mathcal{A} v, u\right\rangle \text { for } v \in D\left(f^{\prime}\right),\langle v, f\rangle=0 \text { for } v \in \mathcal{H}^{s, \boldsymbol{\delta}^{\prime}}\left(\mathbb{R} \times X ; F^{\prime}, G^{\prime}\right) \ominus D\left(f^{\prime}\right),
$$

we can define a continuous operator (23) in the case $\delta_{-}>\delta_{+}$by

$$
\mathcal{A} u:=f
$$

for $f$ associated with $u$ via (27).

Theorem 2.9 Let $f(w)$ have no non-bijectivity points on the lines $I_{\delta_{ \pm}}, \delta_{-}>\delta_{+}$. Then the operator (23) just defined is a Fredholm operator with

$$
\operatorname{dim} \operatorname{ker} \mathcal{A}=\sum_{p \in S_{\left(\delta_{+}, \delta_{-}\right)}} \boldsymbol{n}(f(p)), \operatorname{dim} \operatorname{coker} \mathcal{A}=\sum_{p \in S_{\left(\delta_{+}, \delta_{-}\right)}} \boldsymbol{p}(f(p)),
$$

i.e.,

$$
\text { ind } \mathcal{A}=\sum_{p \in S_{\left(\delta_{+}, \delta_{-}\right)}}\{\boldsymbol{n}(f(p))-\boldsymbol{p}(f(p))\} \text {. }
$$

Example 2.10 Let us consider operator families $\mathcal{A}(\tau)$ of the form (9) for the case $l=0$. Then $\mathcal{A}(\tau)$ extends to an element $\mathcal{A}(w) \in \mathcal{B}^{\boldsymbol{\mu}, 0}(X ; \boldsymbol{v} ; \mathbb{C})$ for $\boldsymbol{v}=\left(1,1,0, \mathbb{C}^{N}\right)$ and a corresponding matrix $\boldsymbol{\mu}$ of orders. Assuming that $\mathcal{A}(\tau)$ is parameter-dependent elliptic with parameter $\tau$ in the sense of Definition 1.1 for $\lambda=\tau \in \mathbb{R}^{1}$, we obtain parameter-dependent ellipticity of $\mathcal{A}(\tau+i \gamma)$ for every $\gamma \in \mathbb{R}$. Applying (15) to $\mathcal{A}(w)$ regarded as an elliptic element of $\mathcal{B}^{\boldsymbol{\mu}, 0}\left(X ; \boldsymbol{v} ; S_{\left(c^{\prime}, c^{\prime \prime}\right)}\right)$ for given $c^{\prime}<c^{\prime \prime}$ we obtain an elliptic $\mathcal{A}_{0}(w) \in \mathcal{B}^{0,0}\left(X ; \boldsymbol{v} ; S_{\left(c^{\prime}, c^{\prime \prime}\right)}\right)$. Applying the Theorems 2.8 and 2.9 we immediately obtain analogous index formulas also for the original Fredholm operators

$$
\mathcal{A}: H^{s, \boldsymbol{\delta}}(\mathbb{R} \times \operatorname{int} X) \rightarrow \begin{gathered}
H^{s-\mu ; \boldsymbol{\delta}}(\mathbb{R} \times \operatorname{int} X) \\
\bigoplus \\
\bigoplus_{j=1}^{N} H^{s-\mu_{j}-\frac{1}{2} ; \boldsymbol{\delta}}(\mathbb{R} \times Y)
\end{gathered} .
$$

Similar relations hold for differential operators in distributional sections of vector bundles.

Remark 2.11 In [2] we apply Theorem 2.8 to specific operator families as in Example 2.10 for the case that $X$ is an interval with $Y$ as the two end points; in these applications the nonbijectivity points of the underlying operator-valued symbol are explicitly known. We employ, in fact, a small modification of the present situation, where we consider weighted cone Sobolev spaces on $\mathbb{R}_{+} \times \operatorname{int} X$ and $\mathbb{R}_{+} \times Y$, respectively, which are linked to the present spaces by a substitution $r=e^{-t}$ which transforms $\mathbb{R}$ to $\mathbb{R}_{+}$.

\section{References}

[1] L. Boutet de Monvel, Boundary problems for pseudo-differential operators, Acta Math. 126 (1971), 11-51.

[2] G. Dines, N. Harutjunjan and B.-W. Schulze, The Zaremba problem in edge Sobolev spaces, (in preparation). 
[3] I.C. Gohberg and E.I. Sigal, An operator generalization of the logarithmic residue theorem and the theorem of Rouché, Math. USSR Sbornik 13, 4 (1971), 603-625.

[4] G. Harutjunjan and B.-W. Schulze, Asymptotics and relative index on a cylinder with conical cross section, Preprint 2002/27, Institut für Mathematik, Potsdam, 2002.

[5] G. Harutjunjan and B.-W. Schulze, Reduction of orders in boundary value problems without the transmission property, Preprint 2002/03, Institut für Mathematik, Potsdam, 2002, Journal of the Math. Soc. of Japan (to appear).

[6] D. Kapanadze and B.-W. Schulze, Crack theory and edge singularities, Kluwer Academic Publ., Dordrecht, 2003, (to appear).

[7] V.A. Kondratyev, Boundary value problems for elliptic equations in domains with conical points, Trudy Mosk. Mat. Obshch. 16 (1967), 209-292.

[8] T. Krainer, On the inverse of parabolic boundary value problems for large times, Preprint 2002/12, Institut für Mathematik, Potsdam, 2002.

[9] R.B. Melrose and G.A. Mendoza, Elliptic operators of totally characteristic type, Preprint MSRI 047 - 83, Math. Sci. Res. Institute, 1983.

[10] S. Rempel and B.-W. Schulze, Index theory of elliptic boundary problems, AkademieVerlag, Berlin, 1982.

[11] E. Schrohe and B.-W. Schulze, Boundary value problems in Boutet de Monvel's calculus for manifolds with conical singularities I, Advances in Partial Differential Equations (Pseudo-Differential Calculus and Mathematical Physics), Akademie Verlag, Berlin, 1994, pp. 97-209.

[12] E. Schrohe and B.-W. Schulze, Boundary value problems in Boutet de Monvel's calculus for manifolds with conical singularities II, Advances in Partial Differential Equations (Boundary Value Problems, Schrödinger Operators, Deformation Quantization), Akademie Verlag, Berlin, 1995, pp. 70-205.

[13] B.-W. Schulze, Corner Mellin operators and reduction of orders with parameters, Ann. Sc. Norm. Sup. Pisa Cl. Sci. 16, 1 (1989), 1-81.

[14] B.-W. Schulze and N.N. Tarkhanov, Euler solutions of pseudodifferential equations, Integral Equations Operator Theory 33 (1999), 98-123.

[15] B.Ju. Sternin, Quasi-elliptic equations in an infinite cylinder, Lecture Notes of the Institute of Electronic Engineering, Moscow, 1973. 